\title{
Preparing ALICE for First Proton-Proton Minimum-Bias Measurements
}

\section{Jan Fiete Grosse-Oetringhaus*}

CERN, 1211 Geneva 23, Switzerland

E-mail: Jan.Fiete.Grosse-Oetringhaus@cern.ch

\begin{abstract}
This publication discusses the status and plans of first minimum-bias measurements in pp collisions with ALICE. It introduces the ALICE experiment with a focus on the subdetectors that are to be used for first physics. The characteristic features of ALICE - the very low-momentum cutoff, the low material budget, and the excellent PID and vertexing capabilities - that make ALICE an important contributor to LHC physics in the realm of soft QCD, are described. A selection of measurements that are accessible with data taken in a few days to 1-2 weeks are discussed: the pseudorapidity density $d N_{c h} / d \eta$, the multiplicity distribution, and the transverse momentum distribution $d N_{c h} / d p_{T}$ of charged particles. The analysis procedures and the associated systematic uncertainties are briefly discussed.
\end{abstract}

XXth Hadron Collider Physics Symposium

November 16 - 20, 2009

Evian, France

\footnotetext{
${ }^{*}$ for the ALICE collaboration

† Speaker.
} 


\section{A Large Ion Collider Experiment (ALICE)}

ALICE [1] is a general-purpose particle detector optimized to study heavy-ion collisions at the LHC. The detector's unique features are high-precision tracking and particle identification in an environment of very high particle densities over a large range of momenta, from tens of $\mathrm{MeV} / c$ to over $100 \mathrm{GeV} / c$, thereby accessing topics starting from soft physics to high- $p_{T}$ particle production and jets. In ALICE it is possible to reconstruct the primary vertex with a resolution of about $100 \mu \mathrm{m}$ in pp collisions and $10 \mu \mathrm{m}$ in $\mathrm{Pb}+\mathrm{Pb}$ collisions. In addition, secondary vertices of e.g. hyperon and heavy quark meson decays can be determined with a resolution of about $100 \mu \mathrm{m}$.

The detector consists of a central barrel part $(|\eta|<0.9)$ optimized for the measurement of hadrons, electrons and photons, a muon spectrometer at forward rapidities $(-2.5<\eta<-4.0)$ as well as additional forward and trigger detectors. The central barrel is contained in a magnetic field of up to $0.5 \mathrm{~T}$. Figure 1 shows the coverage in pseudorapidity $\eta=-\ln \tan \theta / 2$ ( $\theta$ being the angle w.r.t. the beam axis) of the various subdetectors.

Besides the heavy-ion program where ALICE will take data mostly in $\mathrm{Pb}+\mathrm{Pb}$ collisions with up to $\sqrt{s_{\mathrm{NN}}}=5.5 \mathrm{TeV}$, the study of pp collisions is an integral part of the physics program. Due to its acceptance down to very low $p_{T}$, ALICE can measure global event properties well. Furthermore, ALICE will study high-multiplicity pp collisions with a dedicated trigger and measure the charm cross section down to very low $p_{T}$.

\subsection{Silicon Pixel Detector (SPD)}

The Silicon Pixel Detector (SPD), based on hybrid silicon pixel chips, comprises the first two layers of the Inner Tracking System (ITS) that are placed at a radial distance of $3.9 \mathrm{~cm}$ and $7.6 \mathrm{~cm}$ from the beam line. The SPD enables measurements in an environment of 50 particles per $\mathrm{cm}^{2}$ (expected for central $\mathrm{Pb}+\mathrm{Pb}$ collisions) with an occupancy of only a few percent. The SPD allows to access the multiplicity with a low-momentum cut-off of about $35 \mathrm{MeV} / c$ at $0.5 \mathrm{~T}$ field. Particles in $|\eta|<1.4$ emerging from collisions at the nominal interaction-point leave hits in both layers. However, the spread of the $z$ vertex position allows to measure the $d N_{c h} / d \eta$ distribution up to $\eta \approx \pm 2.0$. The pixels themselves have an efficiency of above $99 \%$. However, at present (December 2009) about 17\% of the detector cannot be powered due to problems with the cooling.

Each of the SPD's 1200 chips provides a so-called fast OR signal which indicates if at least one signal was detected in the given chip. This is used for the minimum-bias trigger and allows to trigger on high-multiplicity events.

The SPD modules have been aligned using 50,000 cosmic muons tracks. Fig. 2 shows the track-to-track distance of the reconstructed upper and lower half of traversing cosmic muons before and after alignment. This allows to estimate the effect of the residual misalignment which amounts to less than $10 \mu \mathrm{m}$ in the $r \phi$ direction [2].

\subsection{Time Projection Chamber (TPC)}

ALICE's Time Projection Chamber (TPC), the largest in the world, spans between $0.85 \mathrm{~m}$ and $2.5 \mathrm{~m}$ in radial direction with a length of $5 \mathrm{~m}$. It measures up to 159 clusters per track within $|\eta|<0.9$ which results in an excellent track resolution and enabled good particle identification in the relativistic rise region without being affected by the Landau tail in the energy-loss distribution. 


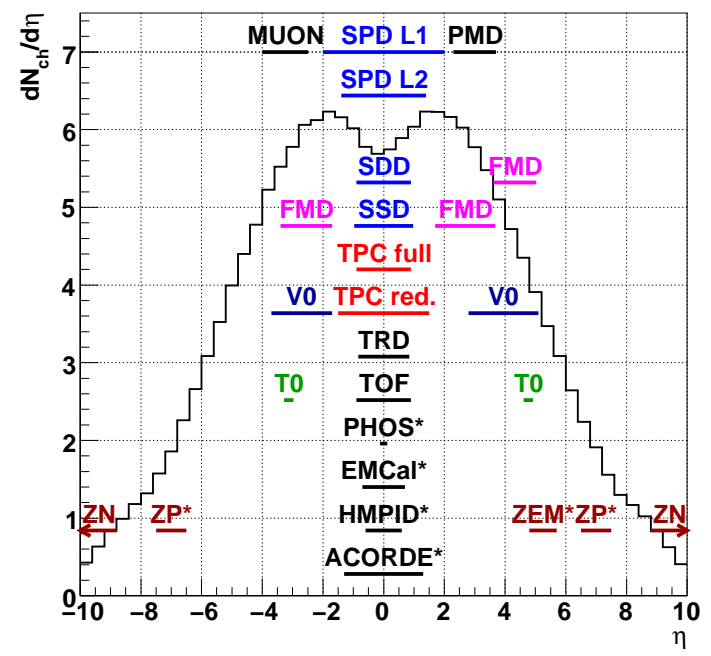

Figure 1: Acceptance in $\eta$ of the subdetectors overlaid with a $d N_{c h} / d \eta$ prediction by Pythia at $\sqrt{s}=$ $14 \mathrm{TeV}$. The subdetectors have full coverage in azimuth except the ones marked with an asterisk. Two ranges are given for the TPC, depending if full or one third track length is required. For more details see [1].

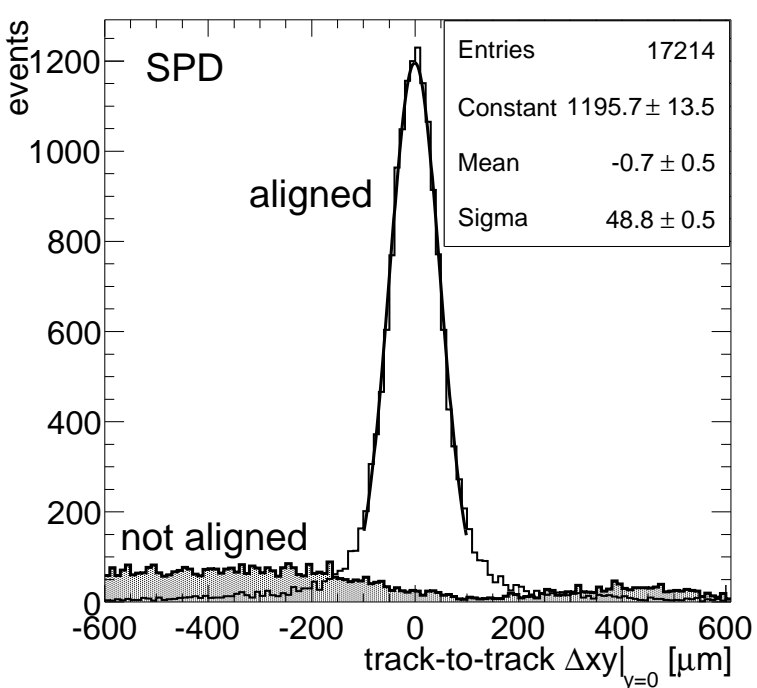

Figure 2: Track-to-track distance of the lower and upper half of cosmic tracks before (shaded histogram) and after alignment (open histogram). Figure from [2].

The low-momentum cut-off is about $150 \mathrm{MeV} / \mathrm{c}$ for pions and $200-300 \mathrm{MeV} / \mathrm{c}$ for other particle species. Above that momentum the geometrical acceptance is about $90 \%$. At present (December 2009) $99.85 \%$ of the channels of the TPC are active for data-taking. A track that traverses ITS and TPC sees only about $13 \%$ of a radiation length.

The physics performance has been assessed using cosmic rays that traverse the TPC and pass close to the beam line. The track parameters of the independent measurement of the lower and upper part of each track are compared. The $p_{T}$ resolution was found to be about $2 \%$ at $p_{T}=$ $2 \mathrm{GeV} / c$ increasing to $4 \%$ at $5 \mathrm{GeV} / c$ (design value: $5 \%$ in a low-multiplicity environment [3]), and $7 \%$ at $10 \mathrm{GeV} / c$. The resolution of the specific energy loss approaches $4.5 \%$ for tracks with more than 140 clusters (design value: $5.5 \%$ [3]).

\subsection{Minimum-Bias Trigger}

The minimum-bias trigger is based on signals from the SPD and V0 detectors. The V0 consists out of two arrays of segmented scintillator counters. The time resolution is better than $1 \mathrm{~ns}$ [1] which allows to identify beam-induced background that occurred outside of the nominal interaction region. Based on cross sections predicted by Pythia [4] and the detector simulation AliRoot [5] taking into account the mentioned hardware status, the MB1 trigger (which requires either a signal in SPD or V0 and no beam-induced background detection) is estimated to select about $86 \%$ of the inelastic cross section at $\sqrt{s}=900 \mathrm{GeV}$. For non single-diffractive (NSD) events it selects about $96 \%$ at $900 \mathrm{GeV}$. For $14 \mathrm{TeV}$ these values are $1-2 \%$ higher. 
For first measurements, the minimum-bias trigger is not used during data-taking, but it is employed for the offline event selection in the analysis. Instead a bunch-crossing trigger will be used that allows to evaluate the trigger performance. Furthermore, a trigger on bunches from one beam only that pass through the experiment allows to estimate the rate of beam-induced background.

\section{Event Characterization at the LHC}

The study of minimum-bias collisions gives important insight into the particle-production mechanism that, although extrapolations can be made from previous measured energies, is unknown at a new energy. In particular it is expected that the majority of the collisions in the energy realm of the LHC feature more than one parton interaction per event that contribute significantly to hadron production. Furthermore, the measurements allow to find a good description of the underlying event which is fundamental for the analysis of rare signals at the LHC. The required tuning of MC generators will be performed using measurements from all LHC experiments. In the specific case of ALICE such measurements are also needed as a baseline for the upcoming heavyion collision measurements. In the subsequent sections selected minimum-bias measurements are presented.

\subsection{Pseudorapidity Density $d N_{c h} / d \eta$}

The measurement of the charged-particle pseudorapidity density will be performed with the SPD. It can also be done with the TPC which allows a cross check in the overlapping phase space region. With about $20,000-40,000$ events a negligible statistical error is achievable. $d N_{c h} / d \eta$ is calculated by counting the number of tracks $(T)$ in a given $\eta$-bin $(d \eta)$ and normalizing with the number of events $(E)$, taking into account a set of corrections $C: d N_{c h} / d \eta=T / E \times C$. One corrects for acceptance, tracking efficiency, and effects of secondaries, decay, and stopping of particles. Furthermore, the efficiency of the vertex reconstruction and the trigger bias has to be corrected for. Collisions occurring at large $|\mathrm{vtx}-z|$ can be used to extend the $\eta$-range, however, the normalization (E) becomes $\eta$-dependent. For the measurement with the TPC an additional correction has to be applied to correct for the low-momentum cut-off. The average correction factors that are expected are about 1.5 for the measurement with the SPD and 1.35 for the measurement with the TPC.

The systematic uncertainties that are associated with this measurement have been evaluated in detail considering uncertainties in the relative cross sections between the different processes (non-diffractive, single-diffractive, and double-diffractive), in the particle composition, and in the $p_{T}$ spectrum. Furthermore, effects of possible uncertainties in the detector geometry (material budget and misalignment) have been studied. The effect of pile-up and beam-induced background is expected to be negligible for the initial LHC running conditions, but this can be only finally assessed considering the actual running conditions during data taking. The total uncertainties are estimated to be less than 5\% for the inelastic sample and less than $10 \%$ for the NSD sample. More details can be found in [6].

Figure 3 (left panel) shows a compilation of $d N_{c h} /\left.d \eta\right|_{\eta=0}$ as function of $\sqrt{s}$ from about $20 \mathrm{GeV}$ to $1.8 \mathrm{TeV}$. Predictions for the expected values at LHC energies are numerous and are summarized e.g. in [7]: for $d N_{c h} /\left.d \eta\right|_{\eta=0}$ these range from 4.3 to 6.4 at $\sqrt{s}=7 \mathrm{TeV}$ and from 4.5 to 7.8 at $14 \mathrm{TeV}$. This is visualized by vertical arrows in the figure. Already an initial measurement 

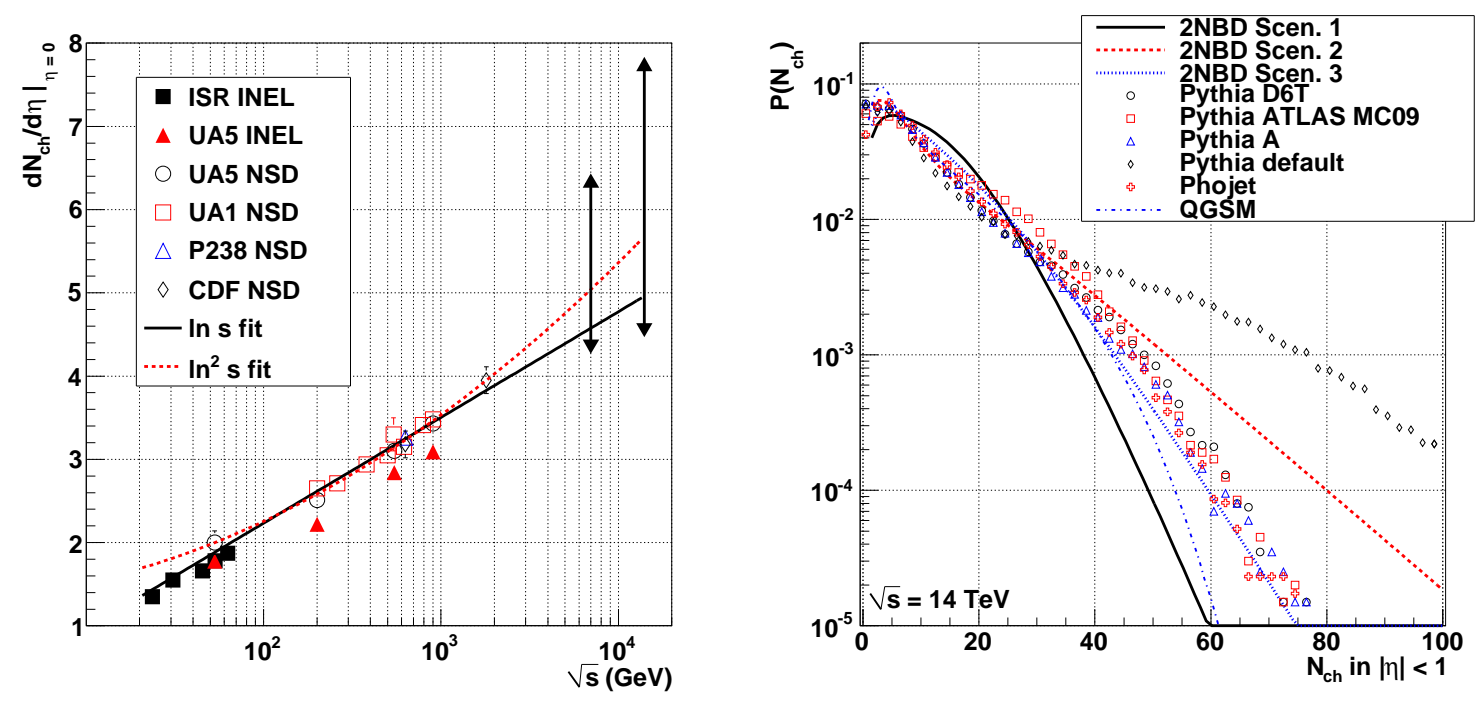

Figure 3: Left panel: $d N_{c h} /\left.d \eta\right|_{\eta=0}$ as function of $\sqrt{s}$. The arrows show the range of predictions (see text). Right panel: Predictions for the multiplicity distribution in $|\eta|<1$ at $14 \mathrm{TeV}$. Figures from [7]. For a description of the data sample and models used, see [7] and the references therein.

with its associated systematic uncertainties will allow to constrain or reject the models that underlie these predictions.

\subsection{Charged-Particle Multiplicity Distribution}

The measurement of the multiplicity distribution is performed with the SPD. 20,000-40,000 events are sufficient to obtain a distribution that reaches up to about 5 times the mean multiplicity. The steeply falling multiplicity spectrum necessitates to unfold the measured data. This allows to correct the multiplicity distribution unaffected by bin flow and independent of the MC input distribution that has been used to determine the detector response. Two unfolding methods have been prepared for the analysis, a method based on $\chi^{2}$ minimization [8] and one based on Bayes' theorem [9]. Both perform well and will be used to obtain the multiplicity distribution from first data. A similar set of systematic uncertainties like discussed in the previous section has been evaluated for the measurement of the multiplicity distribution. The estimated total uncertainties are expected to be less than $9 \%$ (18\%) for the inelastic (NSD) sample. More information about the correction procedure and the performed systematic studies can be found in [6].

Predictions for multiplicity distributions at $\sqrt{s}=14 \mathrm{TeV}$ are shown in Fig. 3 (right panel) for the central region of phase space $(|\eta|<1)$. The predictions differ in the low-multiplicity region by about a factor 2 and by more than an order of magnitude in the high-multiplicity tail. The initial measurements will be able to distinguish between most of the models.

\subsection{Transverse-Momentum Distribution $d N_{c h} / d p_{T}$}

The $p_{T}$ spectrum of charged particles from about $150 \mathrm{MeV} / c$ up to a $p_{T}$ of $10 \mathrm{GeV} / c$ can be obtained from a sample of about 150,000 events using the TPC. The correction procedure is applied in a bin-by-bin fashion similar to the $d N_{c h} / d \eta$ measurement. Unfolding the measured 
distribution is not needed due to the good $p_{T}$-resolution. Loose quality cuts will be applied in the first measurement to be able to use data with an initial calibration and alignment. The estimated total systematic uncertainties for this measurement are expected to be less than $4 \%$ (10\%) for the inelastic (NSD) sample.

$p_{T}$ spectra of identified particles in the region up to $1 \mathrm{GeV} / c$ can be obtained already from 20,000-40,000 events.

Furthermore, it is interesting to study the correlation $\left\langle p_{T}\right\rangle$ vs. $N_{c h}$, the correlation between particle production and transverse energy. This quantity has been measured last at $1.96 \mathrm{TeV}$ [10] and its behavior is only described by very few models or event generators (see e.g. [11]).

\section{Summary}

ALICE can measure within a few days to 1-2 weeks of data-taking basic properties of $\mathrm{pp}$ collisions from $\sqrt{s}=900 \mathrm{GeV}$ to $14 \mathrm{TeV}$. The measurements in this period will allow to access the phase space around mid-rapidity $(|\eta|<2.0)$ from a very low momentum of about $35 \mathrm{MeV} / \mathrm{c}$ to about $10 \mathrm{GeV} / c$. Furthermore, particle identification is available over a large momentum range. These measurements allow to characterize the underlying event, tune MC generators, and in consequence open the door for the study of rare signals and heavy-ion collisions.

The outlined topics compose only the very first measurements available with ALICE. Other interesting topics that will be addressed in pp collisions are baryon transport, strangeness and resonance production, femtoscopy, the study of heavy flavor and jets, and many more.

About a week after this conference, ALICE reported the pseudorapidity density of pp collisions at $\sqrt{s}=900 \mathrm{GeV}$ based on 284 events that were taken during the first LHC run [12].

\section{References}

[1] K. Aamodt et al., JINST 3 (2008) S08002.

[2] ALICE Collaboration, submitted to JINST, arXiv:1001.0502 [physics.ins-det] (2010).

[3] ALICE Collaboration, CERN/LHCC 2000-001 (2000).

[4] T. Sjostrand et al., Comput. Phys. Commun. 135 (2001) 238; V6.4.14 with D6T tune: M.G. Albrow et al. (Tev4LHC QCD Working Group), arXiv:hep-ph/0610012 (2006).

[5] ALICE Collaboration, AliRoot, http://aliceinfo.cern.ch/Offline/

[6] J.F. Grosse-Oetringhaus, PhD thesis, Univ. of Münster, Germany (2009), CERN-THESIS-2009-033.

[7] J.F. Grosse-Oetringhaus and K. Reygers, submitted to J. Phys. G, arXiv:0912.0023 [hep-ex] (2009).

[8] D. Buskulic et al. [ALEPH Collaboration], Z. Phys. C 69 (1995) 15.

[9] G. D’Agostini, Nucl. Instrum. Meth. A 362 (1995) 487.

[10] T. Aaltonen et al. [CDF Collaboration], Phys. Rev. D 79 (2009) 112005 [arXiv:0904.1098 [hep-ex]].

[11] P. Z. Skands, arXiv:0905.3418 [hep-ph] (2009).

[12] ALICE Collaboration, Eur. Phys. J. C 65 (2010) 111 [arXiv:0911.5430 [hep-ex]]. 